\title{
Nasopharyngeal carcinoma with paranasal sinus invasion: the prognostic significance and the evidence-based study basis of its T-staging category according to the AJCC staging system
}

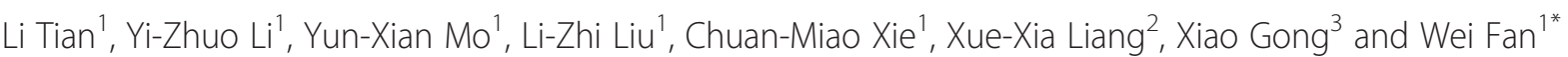

\begin{abstract}
Background: To evaluate the prognostic significance of paranasal sinus invasion for patients with NPC and to provide empirical proofs for the T-staging category of paranasal sinus invasion according to the AJCC staging system for nasopharyngeal carcinoma.
\end{abstract}

Methods: The clinical records and imaging studies of 770 consecutive patients with newly diagnosed, untreated, and nondisseminated NPC were reviewed retrospectively. The overall survival, distant metastasis-free survival, and local relapse-free survival of these patients were analyzed using the Kaplan-Meier method, and the differences were compared using the log-rank test.

Results: The incidence of paranasal sinus invasion was $23.6 \%$, with the rate of incidence of sphenoid sinus invasion being the highest. By multivariate analysis, paranasal sinus invasion was shown to be an independent prognostic factor for overall survival, distant metastasis-free survival, and local relapse-free survival ( $p<0.05$ for all). No significant differences in overall survival, distant metastasis-free survival, and local relapse-free survival were observed between patients with sphenoid sinus invasion alone and those with maxillary sinus and ethmoid sinus invasion $(p=0.87$, $p=0.80$, and $p=0.37$, respectively). The overall survival, distant metastasis-free survival, and local relapse-free survival for patients with stage T3 disease with paranasal sinus invasion were similar to the survival rates for patients with stage T3 disease without paranasal sinus invasion ( $p=0.22, p=0.15$, and $p=0.93$, respectively). However, the rates of overall survival and local relapse-free survival were better for patients with stage T3 disease with paranasal sinus invasion than for patients with stage T4 disease ( $p<0.01$, and $p=0.03$, respectively).

Conclusions: Paranasal sinus invasion is an independent negative prognostic factor for NPC, regardless of which sinus is involved. Our results confirm that it is scientific and reasonable for the AJCC staging system for nasopharyngeal carcinoma to define paranasal sinus invasion as stage T3 disease.

Keywords: Nasopharyngeal carcinoma, Paranasal sinus, Invasion, Prognosis, Staging

\footnotetext{
* Correspondence: fanwei_9798@163.com

'Imaging Diagnosis and Interventional Center, Sun Yat-sen University Cancer

Center; State Key Laboratory of Oncology in South China; Collaborative

Innovation Center for Cancer Medicine, 651 Dongfeng Road East,

Guangzhou 510060, People's Republic of China

Full list of author information is available at the end of the article
} 


\section{Background}

Nasopharyngeal carcinoma (NPC) is endemic in Southeast Asia, especially in the southern provinces of China [1]. The occurrence of paranasal sinus invasion is not unusual, with an incidence of nearly 30\% based on CT and MRI findings [2]. Sphenoid sinus invasion is the most common, followed by maxillary sinus and ethmoid sinus invasion.

The tumor-node-metastasis (TNM) staging system for malignancies is used to evaluate prognosis, aid treatment planning, and facilitate the stratification of treatment. At present, the seventh edition of the American Joint Committee on Cancer (AJCC) staging system is widely used throughout the world, and patients with NPC and paranasal sinus invasion are defined as stage $\mathrm{T} 3$ according to the staging system [3]. With regard to the prognostic value of paranasal sinus invasion and its suitable position in the $\mathrm{T}$ staging, there are few literature reports for reference. Tao et al. developed a prognostic scoring system (PSS) that could help identify NPC patients with different risk for locoregional relapse, and found that sphenoid sinus, ethmoid sinus and maxillary sinus invasion were classified as different risk groups [4]. While Mao et al. considered sphenoidal sinus invasion alone had a better outcome for patients with NPC than did other paranasal sinus invasion [5]. Both studies indicated that tumor invasion into the different paranasal sinuses might have different effects on the prognosis of patients with NPC. On the other hand, the results of Pan et al. revealed that when paranasal sinus invasion were classified as T3 according the 7th edition AJCC $\mathrm{T}$ classification, the segregation of LRFS curves between stage T3 and T4 groups could be well displayed [6]. Which, in a sense, have provided evidence and reference for the AJCC T- staging.

In the present staging system for NPC, radiologic imaging, especially MRI, plays an important role. In comparison to CT, MRI, with its superior soft-tissue contrast, can provide a more accurate definition of early invasion beyond the nasopharynx and a more accurate assessment of the parapharyngeal space, skull base, paranasal sinus, and cranial nerve invasion [7-9]. Given these advantages, MRI is considered the optimal imaging technique for studying the extension of local disease in NPC.

Therefore, we conducted a retrospective study with a large sample size to evaluate the prognostic significance of paranasal sinus invasion for patients with NPC and its suitable position in the $\mathrm{T}$ classification, and thus to provide more empirical proofs for the AJCC staging system.

\section{Methods}

Patient population

This retrospective study was approved by the Institutional Review Board of Sun Yat-Sen University, Guangzhou, China. Between December 2003 and December 2005, 782 consecutive patients with newly diagnosed, untreated, and nondisseminated NPC were recruited for this study. Of the 782 patients, 12 were subsequently eliminated from the study, including nine patients who were unable to complete radiation therapy and three patients in whom new pulmonary nodules and hepatic lesion were detected when the first course of treatment just started. The remaining 770 patients were included in our retrospective study. The median age of the patients was 44 years (range, 13-75 years), with a male-to-female ratio of 3.3:1. All of the patients underwent a pretreatment evaluation that included a complete patient history, physical and neurologic examinations, hematologic and biochemistry profiles, whole MR imaging of the neck and nasopharynx, chest radiography, and abdominal ultrasonography. A total of 225 patients with stage N2 or N3 disease underwent emission computed tomography (ECT), and 32 of the 770 patients $(4.2 \%)$ underwent positron emission tomography-CT. The patients' medical records and imaging studies were analyzed retrospectively, and the NPC stage was classified according to the seventh edition of the AJCC staging system [3]. The characteristics of the 770 patients are shown in Table 1.

\section{Table 1 The characteristics of $\mathbf{7 7 0}$ patients with} nasopharyngeal carcinoma

\begin{tabular}{lc}
\hline Characteristics & Number of patients(\%) \\
\hline Sex & \\
Male & $590(76.6 \%)$ \\
Female & $180(23.4 \%)$ \\
Age & \\
$\geq 50$ years old & $255(33.1 \%)$ \\
$<50$ years old & $515(69.9 \%)$ \\
Histologic type & \\
WHO II/III & $755(98.1 \%)$ \\
WHO I & $15(1.9 \%)$ \\
T classification & \\
T1 & $121(15.7 \%)$ \\
T2 & $115(14.9 \%)$ \\
T3 & $346(44.9 \%)$ \\
T4 & $188(24.5 \%)$ \\
N classification & \\
NO & $83(10.8 \%)$ \\
N1 & $462(60.0 \%)$ \\
N2 & $203(26.4 \%)$ \\
N3 & $22(2.8 \%)$ \\
Stage & \\
I & $120(15.6 \%)$ \\
II & $234(30.4 \%)$ \\
III & $302(39.2 \%)$ \\
IVA IVB & $114(14.8 \%)$ \\
\hline
\end{tabular}




\section{MR imaging protocol and image assessment}

All patients underwent MR imaging with a 1.5 -T system (Signa CV/i; GE Healthcare, Chalfont St Giles, England). The region from the suprasellar cistern to the inferior margin at the sternal end of the clavicle was examined with a head-and-neck coil. T1-weighted, fast spin-echo images in the axial, coronal, and sagittal planes (repetition time msec/echo time msec, 500-600/10-20) and T2-weighted, fast spin-echo MR images in the axial plane (4000-6000/ 95-110) were obtained before the injection of contrast material. After intravenous administration of gadopentetate dimeglumine (Magnevist; Schering, Berlin, Germany) at a dose of $0.1 \mathrm{mmol}$ per kilogram of body weight, the axial and sagittal T1-weighted spin-echo sequences and coronal T1-weighted fat-suppressed spin-echo sequences were performed sequentially using the same parameters applied prior to the injection of gadopentetate dimeglumine. A section thickness of $5 \mathrm{~mm}$, an intersection gap of $1 \mathrm{~mm}$ and a matrix of $512 \times 512$ were used.
All MR images were reviewed by two radiologists with more than 10 years of experience in MR imaging of head and neck cancers. All images were evaluated independently, and disagreements were resolved by consensus. Diagnostic MRI criteria for the invasion of the paranasal sinuses included the following: (1) tumors that had invaded into the sinus cavity connected with a primary nasopharyngeal lesion and with bone destruction of the wall of the sinus (Figure 1) and (2) presentation with an equal or lower signal in the T1WI MRI scan, an equal or higher signal in the T2WI and an obvious enhancement in the enhanced MRI scan, with the same signal intensity characteristics as revealed in the primary lesion $[2,10]$.

\section{Treatment}

All patients were treated with definitive-intent radiation therapy. A total of 618 of the 770 patients (80.2\%) underwent two-dimensional conventional radiation therapy, 115 (14.9\%) underwent intensity-modulated radiation therapy

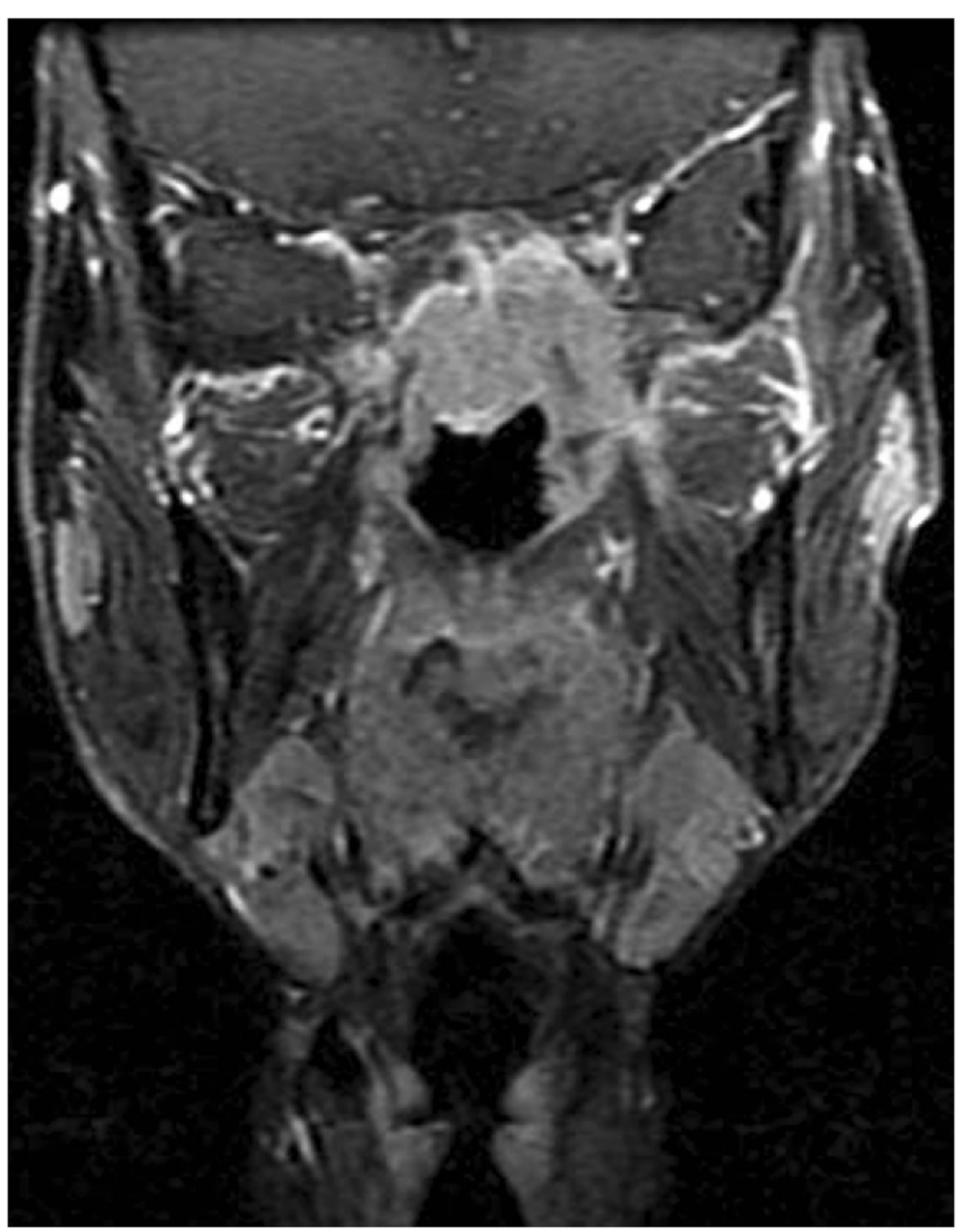

Figure 1 Image of tumor invasion into the sphenoid sinus in patient with NPC. A contrast enhanced coronal T1-weighted MR image revealed that the mass connected with the primary nasopharyngeal lesion invaded into the sphenoid sinus and that the floor of the sphenoid sinus was destroyed. 
(IMRT), and 37 (4.8\%) underwent three-dimensional conformal radiation therapy. Details regarding the radiation therapy techniques have been reported previously [11-13].

Of the 416 patients with stage III or IV NPC (classified as stage T3-T4 and/or stage N2-N3), 370 (89\%) received neoadjuvant, concomitant, or adjuvant chemotherapy. When possible, salvage treatments, including afterloading, surgery, and chemotherapy, were provided in the event of documented relapse or if the disease persisted.

\section{Follow up}

The follow-up period was estimated from the first day of treatment to either the day of death or the day of the last examination. Follow up was performed with imaging or clinical assessment. The patients were evaluated at least once every three months during the first two years; thereafter, patients were followed up every six months until death.

\section{Statistical analysis}

The Statistical Package for Social Sciences 15.0 (SPSS, Chicago, IL) was used for statistical analysis. The following endpoints (interval to the first defining event) were assessed: overall survival (OS), distant metastasis-free survival (DMFS), and local relapse-free survival (LRFS). The actual rates were calculated using the Kaplan-Meier method, and the differences were compared with the log-rank test [14]. Multivariate analyses with the Cox proportional hazards model were used to test independent significance by the backward elimination of insignificant explanatory variables [15]. The criterion for statistical significance was set at $\alpha=0.05$, and $p$ values were based on two-sided test results.

\section{Results}

\section{Incidence of paranasal sinus invasion}

The incidence of paranasal sinus invasion was $23.6 \%$ (182 of 770 patients), with invasion of the sphenoid sinus, maxillary sinus and ethmoid sinus in 162 (21.0\%), 86 (11.2\%) and 38 (4.9\%) of the 770 patients, respectively. None of the patients had frontal sinus invasion. The incidence of sphenoid sinus invasion was higher than that of maxillary sinus and ethmoid sinus invasion in patients with NPC. Of the 162 patients with sphenoid sinus invasion, 89 (54.9\%) did not have maxillary sinus and ethmoid sinus invasion. In contrast, of the 86 patients with maxillary sinus invasion and 38 patients with ethmoid sinus invasion, $69(80.2 \%)$ and $32(84.2 \%)$ also had sphenoid sinus invasion, respectively. Of the 182 patients with paranasal sinus invasion, 97 (53.3\%) had stage T3 disease, and 85 (46.7\%) had stage T4 disease.

\section{Prognosis of patients with paranasal sinus invasion}

The median follow-up period was 84 months (range, 3120 months). In total, 59 patients (7.6\%) developed local- regional failure, 129 patients (16.8\%) developed distant metastases, and 184 patients (23.9\%) died. The 5-year overall survival, distant metastasis-free survival, and local relapse-free survival rates for the entire patient population were $80.2 \%, 84.7 \%$, and $92.4 \%$, respectively. Significant differences were observed between patients without and with paranasal sinus invasion in overall survival (84.4\% vs $67.2 \%, P<0.01$ ), distant metastasis-free survival (88.4\% vs $72.3 \%, P<0.01$ ) and local relapse-free survival (94.0\% vs $87.0 \%, P<0.01$ ), with better outcomes associated with patients without paranasal sinus invasion.

The following parameters, which could possibly influence the prognosis, were included in the Cox proportional hazards model for multivariate analysis: age ( $\geq 50$ years and $<50$ years), sex, nasal cavity extension, oropharyngeal extension, parapharyngeal space extension, skull base erosion, paranasal sinus extension, hypopharyngeal extension, orbit extension, masticator space extension, cranial nerve palsy and intracranial extension, $\mathrm{N}$ classification, use of chemotherapy, radiation therapy technique. The $\mathrm{N}$ classification was treated as ordinary variable in the multivariate analysis. Using multivariate analysis, paranasal sinus invasion was identified as an independent prognostic factor for overall survival, distant metastasis-free survival, and local relapse-free survival $(\mathrm{P}<0.05$ for all). The parapharyngeal space extension and $\mathrm{N}$ classification were found to be independent prognostic factors for both overall survival and distant metastasis-free survival (Table 2).

\section{T-staging category of paranasal sinus invasion}

A total of 182 patients developed paranasal sinus invasion. Owing to the proximity of the floor of the sphenoid

Table 2 Multivariate analysis of prognostic factors for patients with nasopharyngeal carcinoma

\begin{tabular}{lcc}
\hline Endpoint and Variable & $\boldsymbol{P}$ Value & $\begin{array}{c}\text { Odds Ratio (95\% } \\
\text { confidence interval) }\end{array}$ \\
\hline Overall survival & $<0.01$ & $1.80(1.35,2.42)$ \\
Age & $<0.01$ & $1.76(1.28 .2 .42)$ \\
Paranasal sinus involvement & $<0.01$ & $1.95(1.31,2.90)$ \\
Parapharyngeal space extension & $<0.01$ & $1.74(1.27,2.40)$ \\
Intracranial extension & 0.02 & $0.69(0.51,0.93)$ \\
N classification & & \\
Distant metastasis-free survival & $<0.01$ & $1.75(1.23,2.48)$ \\
Age & 0.02 & $1.58(1.08,2.30)$ \\
Paranasal sinus involvement & 0.04 & $1.62(1.02,2.58)$ \\
Parapharyngeal space extension & $<0.01$ & $2.73(1.45,5.16)$ \\
Skull base erosion & $<0.01$ & $0.63(0.44,0.89)$ \\
N classification & & \\
Local relapse-free survival & 0.02 & $1.91(1.11,3.27)$ \\
Paranasal sinus involvement & &
\end{tabular}


sinus to the roof of the nasopharynx, and the majority of patients with maxillary sinus or ethmoid sinus invasion accompanied with sphenoid sinus invasion simultaneously, the 182 patients were divided into two groups. Group 1 was composed of patients with invasion of the sphenoid sinus alone, without invasion of the maxillary sinus and ethmoid sinus, and group 2 was composed of patients with invasion of the maxillary sinus and/or ethmoid sinus. Of the 182 patients with paranasal sinus invasion, 89 and 93 had group 1 and group 2 invasion, respectively. No significant differences in overall survival, distant metastasis-free survival and local relapsefree survival were observed between the patients with group 1 and group 2 invasion ( $p=0.87, P=0.80$, and $p=0.37$, respectively, Figure 2).

According to the seventh AJCC staging system, 346 patients belonged to stage T3, of which, 249 did not develop paranasal sinus invasion (T3a) and 97 developed paranasal sinus invasion (T3b). No significant differences in overall survival, distant metastasis-free survival and local relapse-free survival were observed between patients with T3a and those with T3b $(\mathrm{p}=0.22, \mathrm{p}=0.15$, and $p=0.93$, respectively). However, the rates of overall survival and local relapse-free survival were better for patients with T3b than for patients with stage T4 disease $(\mathrm{p}<0.01$, and $\mathrm{p}=0.03$, respectively) (Figure 3 ). No significant difference in distant metastasis-free survival was observed between patients with $\mathrm{T} 3 \mathrm{~b}$ and those with $\mathrm{T} 4$ disease $(p=0.10)$. When paranasal sinus invasion was classified as stage T3, the segregation of survival curves between the T3 and T4 groups was clearly displayed.

\section{Discussion}

\section{The incidence of paranasal sinus invasion of patients} with NPC

NPC is an aggressive neoplasm, and the spread of the tumor into the paranasal sinuses occurs relatively frequently. The result of the present study, based on the data from a large cohort, suggested that the incidence of the invasion of the paranasal sinus in patients with NPC was $23.6 \%$. The highest rate of incidence was of sphenoid sinus invasion (21\%), followed by maxillary sinus invasion (11.2\%) and ethmoid sinus invasion (4.9\%). Chong et al. reported the CT and MRI findings of 114 patients with NPC, $21 \%, 9 \%$ and $4 \%$ of those patients were detected with sphenoid sinus, maxillary sinus and ethmoid sinus invasion, respectively [2]. While the results of King et al. showed that the incidence rates of sphenoid sinus, maxillary sinus and ethmoid sinus invasion were $27 \%, 5 \%$ and $14 \%$, respectively [16]. Our results are roughly the same as those of Chong et al., but a little different from those of King et al. For patients with NPC, the local disease spreads in a stepwise manner from proximal to distal sites [7]. Tumors of the roof of the nasopharynx tend to spread directly and superiorly into the skull base, where there is no muscle or fascia to act as a barrier against tumor invasion, as well as to the floor of the sphenoid sinus, which borders the nasopharynx roof. Therefore, for most patients with NPC, the primary tumor originating from the nasopharynx directly destroys the floor of the sphenoid sinus. This accounts for the high rate of incidence of sphenoid sinus invasion. Additionally, the posterior wall of the maxillary sinus is adjacent to the pterygopalatine fossa and accordingly, tumors extending anteriorly to the pterygopalatine fossa can easily spread to the maxillary sinus. In contrast, tumors extending to the sphenoid sinus or nasal cavity are likely to invade the ethmoid sinus anteriorly or superiorly.

\section{The prognostic significance of paranasal sinus invasion for patients with NPC}

Our retrospective study, based on large number of cases, revealed that the invasion of the paranasal sinus was an independent negative prognostic factor for overall survival, distant metastasis-free survival, and local relapsefree survival in patients with NPC. Hence, it is scientific and reasonable for paranasal sinus invasion to be included
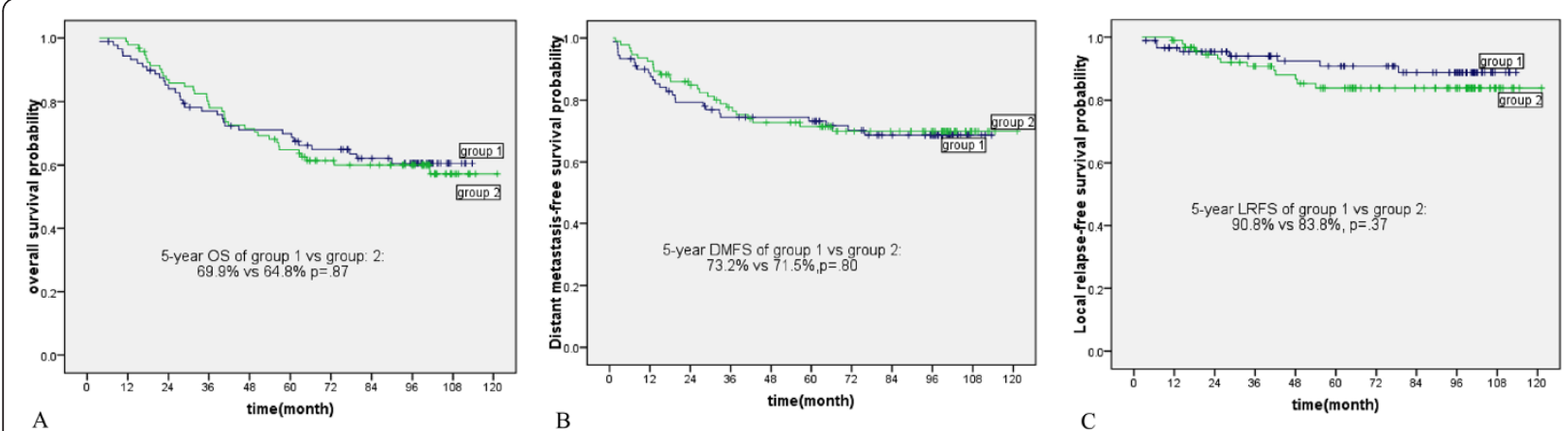

Figure 2 Survival curves of patients with NPC and different paranasal sinus invasion. The graph shows (A) the overall survival probability, (B) the distant metastasis-free survival probability, and (C) the local relapse-free survival probability for patients with sphenoid sinus invasion alone and patients with maxillary sinus and ethmoid sinus invasion. Group 1 and Group 2 represent patients with NPC with sphenoid sinus invasion alone and with maxillary sinus and ethmoid sinus invasion, respectively. 

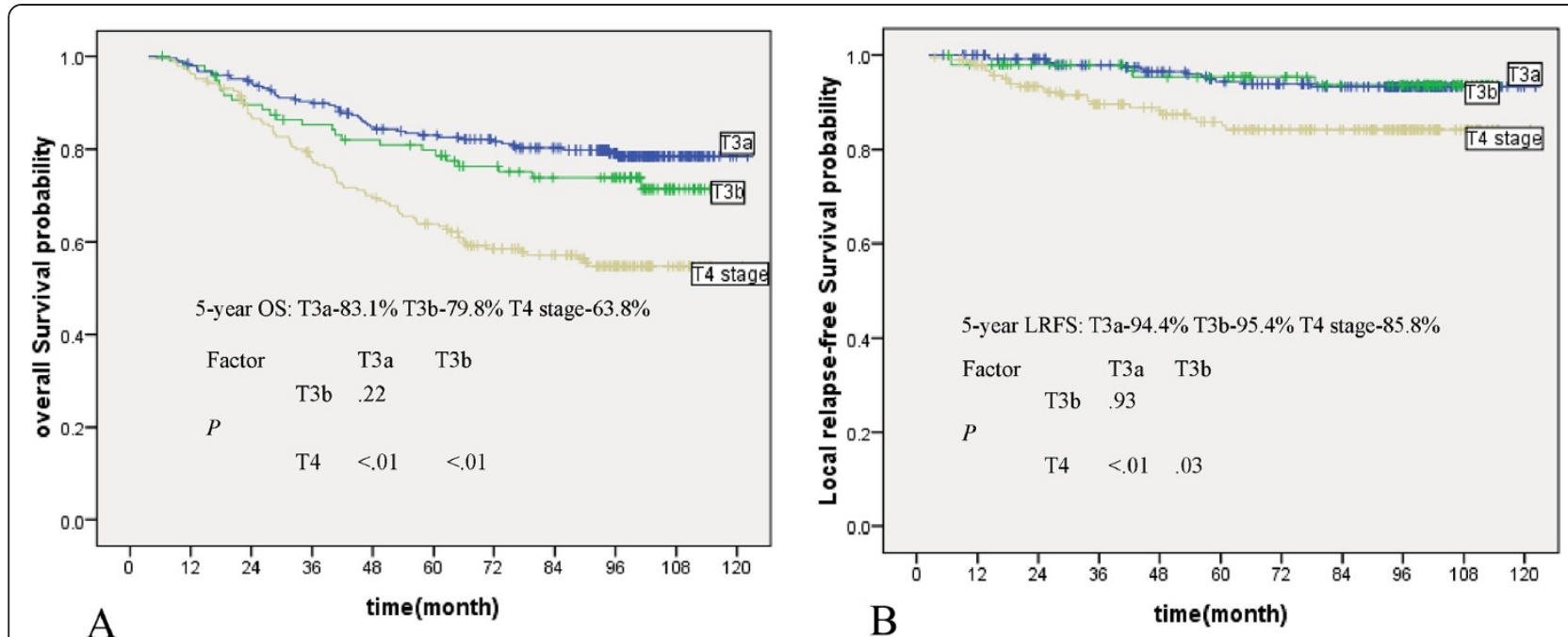

Figure 3 Survival curves of patients with NPC with T3 and T4 disease. The graph shows (A) the overall survival probability and (B) the local relapse-free survival probability for patients with stage T3 and T4 disease according to the seventh AJCC staging system. T3a and T3b represent patients with $\mathrm{T3}$ disease without and with paranasal sinus invasion, respectively.

in the AJCC staging system. Some researchers proposed that the maximum primary tumor diameter (MPTD) or primary tumor volume (PTV) had some effect on the prognosis of patients with NPC [17-21]. Due to the proximity of the floor of the sphenoid sinus to the roof of the nasopharynx, and the majority of patients with maxillary sinus or ethmoid sinus invasion accompanied with sphenoid sinus invasion simultaneously, we had speculated that the primary tumor volume of patients with sphenoid sinus invasion might be a little smaller than that of patients with ethmoid sinus and maxillary sinus invasion, making for somewhat better prognosis for patients. However, the results showed that the prognostic significances for both groups were not significantly different. We speculate this may be due to the following reasons. First, the primary treatment modality for patients with NPC was radiation therapy. With the aid of MRI, the range of local lesions could be evaluated with greater accuracy and the target and field could be designed more rationally [22]. Additionally, the improved treatment strategies for T3-4 patients with NPC, including the boost technique of two-dimensional radiation therapy, IMRT, and the combination of chemotherapy with radiotherapy, have dramatically improved the treatment outcome with respect to loco-regional control $[12,23,24]$. This may be the reason why no difference was observed in the LRFS between patients with sphenoid sinus invasion alone and those with maxillary sinus and ethmoid sinus invasion. Second, as for the DMFS rate, we think the possible reason may lie in the fact that the difference of the tumor volume resulted from the different paranasal sinus invasion may not be significant enough to lead to significant difference of risks for distant failure. For the reasons given above, it is reasonable to consider paranasal sinus invasion as a single entity in the TNM classification, regardless of which sinus is involved.

\section{The evidence-based study basis of T-staging category of paranasal sinus invasion}

In the fifth edition of the AJCC staging system for NPC, patients with paranasal sinus invasion were defined as $\mathrm{T} 3$, and this classification remains in the sixth and the current seventh edition of the AJCC staging system. While paranasal sinus invasion is classified as stage T4 disease according to the Chinese 2008 staging system [25]. Pan et al. compared the predictive value of both staging systems for patients with NPC [6]. The results revealed that for the Chinese 2008 T classification, the 5-year LRFS rates of T3 and T4 groups did not differ significantly, while the rates between both groups were remarkably different according to the 7th edition AJCC T classification. The possible reason lie in that when compared to cranial nerve palsy and intracranial extension, paranasal sinus invasion maybe occur a little bit earlier, accordingly, the tumor volume may be somewhat smaller, which possibly makes for a better prognosis. In a sense, this study demonstrated that it was more suitable for paranasal sinus invasion to be classified as stage T3. Our results, based on a large number of samples, further confirmed this viewpoint, providing more empirical proofs for the rationality of the AJCC T staging.

\section{The influence of MRI on patients with NPC}

All of the patients in the current study were evaluated by MRI. For the lesions of the paranasal sinus in patients with NPC, many inflammatory changes overlapped 
with the neoplastic process, and it is important to differentiate between the two entities and to define the inflammation-tumor border. MR imaging, especially T2weighted and contrast enhanced MR, can help us to solve this challenging problem. The inflammatory lesions usually present high signal intensity on T2-weighted image and a thin superficial enhancement after contrast administration, while the tumors reveal relatively lower signal intensity on T2-weighted image and solid enhancement with contrast administration. In addition, most importantly, the signal intensity and enhancement pattern of tumors invading the paranasal sinus are usually in accordance with those of the primary tumor of the nasopharynx $[2,26,27]$. Over the past several decades, MRI has been used to assess the extent of NPC more reliably and accurately compared with $\mathrm{CT}$, which has been shown to influence the stage assignment and disease prognosis [28-30].

\section{Limitations of this study}

It should be stressed that because of limited resources, most of patients $(80.2 \%)$ in this study were treated with conventional radiotherapy technique. Recently, intensitymodulated radiotherapy (IMRT) has gradually replaced two-dimensional conventional radiotherapy as the primary radiotherapy technique for NPC and has been reported to provide encouraging treatment outcome [31-35]. Therefore, the suitability of the staging system of NPC amid the changes in therapeutic methods needs continual assessment. As well, the effect of paranasal sinus invasion on the prognosis and staging of patients with NPC should be further confirmed by clinic studies.

\section{Conclusion}

Paranasal sinus invasion is an independent negative prognostic factor for NPC, regardless of which sinus is involved. It is scientific and reasonable for paranasal sinus invasion to be defined as stage T3 disease, as proposed in the AJCC staging system. Our study provided some empirical proofs for the AJCC T staging.

\section{Competing interests}

The authors indicate that no actual or potential conflicts of interest exist.

\section{Authors' contributions}

LT and Y-ZL participated in literature research, study design, data collection data analysis, interpretation of findings and the draft of the manuscript. $Y-X M, L-Z L$ and $X-X L$ carried out the data collection. $L-Z L$ and $C-M X$ reviewed MR images. XG performed the statistical analysis. WF contributed with study design, data collection, interpretation of findings and critical edit of the manuscript. All authors read and approved the final manuscript.

\section{Acknowledgements}

The study was granted from the National Natural Science Foundation of China(No. 30371620), and Sci-Tech Research Foundation of Guangzhou City (No.2008J-C141-3)

\section{Author details}

'Imaging Diagnosis and Interventional Center, Sun Yat-sen University Cancer Center; State Key Laboratory of Oncology in South China; Collaborative Innovation Center for Cancer Medicine, 651 Dongfeng Road East,

Guangzhou 510060, People's Republic of China. ${ }^{2}$ Department of Radiation Oncology, Sun Yat-sen University Cancer Center; State Key Laboratory of Oncology in South China; Collaborative Innovation Center for Cancer Medicine, 651 Dongfeng Road East, Guangzhou 510060, People's Republic of China. ${ }^{3}$ Department of Medical Statistics and Epidemiology, School of Public Health, Sun Yat-sen University, 74 Zhongshan Road Second, Guangzhou 510080, People's Republic of China.

Received: 8 February 2014 Accepted: 30 October 2014

Published: 18 November 2014

\section{References}

1. Gu MF, Liu LZ, He LJ, Yuan WX, Zhang R, Luo GY, Xu GL, Zhang HM, Yan

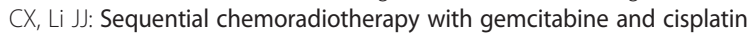
for locoregionally advanced nasopharyngeal carcinoma. Int J Cancer 2013, 132:215-223.

2. Chong VF, Fan YF, Khoo JB: Computed tomographic and magnetic resonance imaging findings in paranasal sinus invasion in nasopharyngeal carcinoma. Ann Acad Med Singapore 1998, 27:800-804.

3. Edge SB, Compton CC: The American Joint Committee on Cancer: the 7th edition of the AJCC cancer staging manual and the future of TNM. Ann Surg Oncol 2010, 17:1471-1474.

4. Tao CJ, Liu X, Tang LL, Mao YP, Chen L, Li WF, Yu XL, Liu LZ, Zhang R, Lin AH, Ma J, Sun Y: Prognostic scoring system for locoregional control among the patients with nasopharyngeal carciinoma treated by intensity-modulated radiotherapy. Chin J Cancer 2013, 32:494-501.

5. Mao YP, Hong MH, Sun Y, Liang SB, Li L, Liu LZ, Tang LL, Cao SM, Lin AH, Lu TX, Liu MZ, Ma J: Clinical staging of nasopharyngeal carcinoma based on MRI: suggestions for improving the Chinese ' 92 staging system. Ai Zheng 2007, 26:1099-1106 [Article in Chinese].

6. Pan J, Xu Y, Qiu S, Zong J, Guo Q, Zhang Y, Lin S, Lu JJ: A comparison between the Chinese 2008 and the 7th edition AJCC staging systems for nasopharyngeal carcinoma. Am J Clin Oncol 2013. Epub ahead of print

7. Liang SB, Sun Y, Liu LZ, Chen Y, Chen L, Mao YP, Tang LL, Tian L, Lin AH, Liu MZ, Li L, Ma J: Extension of local disease in nasopharyngeal carcinoma detected by magnetic resonance imaging: improvement of clinical target volume delineation. Int J Radiat Oncol Biol Phys 2009, 75:742-750.

8. King AD, Vlantis AC, Bhatia KS, Zee BC, Woo JK, Tse GM, Chan AT, Ahuja AT: Primary nasopharyngeal carcinoma: diagnostic accuracy of MR imaging versus that of endoscopy and endoscopic biopsy. Radiology 2011, 258:531-537.

9. Liao XB, Mao YP, Liu LZ, Tang LL, Sun Y, Wang Y, Lin AH, Cui CY, Li L, Ma Ja How does magnetic resonance imaging influence staging according to AJCC staging system for nasopharyngeal carcinoma compared with computed tomography? Int J Radiat Oncol Biol Phys 2008, 72:1368-1377.

10. Chen LS, Wei GY, Hu XF, Zeng XH, Lu QX: Study of MR image for invasion of paranasal sinuses in 56 cases with nasopharyngeal carcinoma. Chin Ger J Clin Oncol 2009, 8:719-721.

11. Luo W, Deng X-W, Lu T-X: Dosimetric evaluation for three dimensional radiotherapy plans for patients with early nasopharyngeal carcinoma. Ai Zheng 2004, 23:605-608 [in Chinese].

12. Zhao C, Han F, Lu LX, Huang SM, Lin CG, Deng XW, Lu TX, Cui NJ: Intensity modulated radiotherapy for local-regional advanced asopharyngeal carcinoma. Ai Zheng 2004, 23:1532-1537 [in Chinese].

13. Ma J, Liu L, Tang L, Zong J, Lin A, Lu T, Cui N, Cui C, Li L: Retropharyngeal lymphadenopathy in nasopharyngeal carcinoma: prognostic value and staging categories. Clin Cancer Res 2007, 13:1445-1452.

14. Kaplan EL, Meier P: Nonparametric estimation from incomplete observations. J Am Stat Assoc 1958, 53:457-481.

15. Cox DR: Regression models and life tables. J R Stat Soc Series B Stat Methodol 1972, 34:187-220.

16. King AD, Lam WW, Leung SF, Chan YL, Teo P, Metreweli C: MRI of local disease in nasopharyngeal carcinoma: tumour extent vs tumour stage. Br J Radiol 1999, 72:734-741.

17. Liang SB, Deng YM, Zhang N, Lu RL, Zhao H, Chen HY, Li SE, Liu DS, Chen Y: Prognostic significance of maximum primary tumor diameter in nasopharyngeal carcinoma. BMC Cancer 2013, 13:260-267. 
18. Lee CC, Ho HC, Lee MS, Hsiao SH, Hwang JH, Hung SK, Chou P: Primary tumor volume of nasopharyngeal carcinoma: significance for survival. Auris Nasus Larynx 2008, 35:376-380.

19. Wu Z, Zeng RF, Su Y, Gu MF, Huang SM: Prognostic significance of tumor volume in patients with nasopharyngeal carcinoma undergoing intensity-modulated radiation therapy. Head Neck 2013, 35:689-694.

20. Shen C, Lu JJ, Gu Y, Zhu G, Hu C, He SC: Prognostic impact of primary tumor volume in patients with nasopharyngeal carcinoma treated by definitive radiation therapy. Larynnoscope 2008, 18:1206-1210.

21. Chen C, Fei Z, Pan J, Bai P, Chen L: Significance of primary tumor volume and T-stage on prognosis in nasopharyngeal carcinoma treated with intensity-modulated radiation therapy. Jpn J Clin Oncol 2011, 41:537-542

22. Chen L, Liu LZ, Mao YP, Tang LL, Sun Y, Chen Y, Lin AH, Li L, Ma J: Grading of MRI-detected skull-base invasion in nasopharyngeal carcinoma and its prognostic value. Head Neck 2011, 33:1309-1314.

23. Liu L, Liang S, Li L, Mao Y, Tang L, Tian L, Liao X, Cui C, Lin A, Ma J: Prognostic impact of magnetic resonance imaging-detected cranial nerve invasion in nasopharyngeal carcinoma. Cancer 2009, 115:1995-2003.

24. Ma J, Mai HQ, Hong MH, Min HQ, Mao ZD, Cui NJ, Lu TX, Mo HY: Results of a prospective randomized trial comparing neoadjuvant chemotherapy plus radiotherapy with radiotherapy alone in patients with locoregionally advanced nasopharyngeal carcinoma. J Clin Oncol 2001 19:1350-1357

25. Committee of Chinese Clinical Staging of Nasopharyngeal Carcinoma: Report on the revision of nasopharyngeal carcinoma '92 staging. Chin J Radiat Oncol 2009, 18:2-6 [in Chinese].

26. Lanzieri CF, Shah M, Krauss MD, Lavertu PL: Use of gadoliniumenhanced MR imaging for differentiating mucoceles from neoplasms in the paranasal sinuses. Radiology 1991, 178:425-428.

27. Som PM, Shapiro MD, Biller HF, Sasaki C, Lawson W: Sinonasal tumors and inflammatory tissues: differentiation with MRI. Radiology 1988, 167:803-807.

28. Chung NN, Ting LL, Hsu WC, Lui LT, Wang PM: Impact of magnetic resonance imaging versus $C T$ on nasopharyngeal carcinoma: primary tumor target delineation for radiotherapy. Head Neck 2004, 26:241-246.

29. Abdel Khalek Abdel Razek A, King A: MRI and CT of nasopharyngeal carcinoma. AJR Am J Roentgenol 2012, 198:11-18.

30. Lee AW, Sze WM, Au JS, Leung SF, Leung TW, Chua DT, Zee BC, Law SC, Teo PM, Tung SY, Kwong DL, Lau WH: Treatment results for nasopharyngeal carcinoma in the modern era: the Hong Kong experience. Int J Radiat Oncol Biol Phys 2005, 61:1107-1116.

31. Sun Y, Tang LL, Chen L, Li WF, Mao YP, Liu LZ, Lin AH, Li L, Ma J: Promising treatment outcomes of intensity modulated radiation therapy for nasopharyngeal carcinoma patients with NO disease according to the seventh edition of the AJCC staging system. BMC Cancer 2012, 12:68.

32. Tham IW, Hee SW, Yeo RM, Salleh PB, Lee J, Tan TW, Fong KW, Chua ET, Wee JT: Treatment of nasopharyngeal carcinoma using intensity modulated radiotherapy-the national cancer centre Singapore experience. Int J Radiat Oncol Biol Phys 2009, 75:1481-1486.

33. Lee N, Xia P, Quivey JM, Sultanem K, Poon I, Akazawa C, Akazawa P, Weinberg V, Fu KK: Intensity modulated radiotherapy in the treatment of nasopharyngeal carcinoma: an update of the UCSF experience. Int J Radiat Oncol Biol Phys 2002, 53:12-22.

34. Kam MK, Teo PM, Chau RM, Cheung KY, Choi PH, Kwan WH, Leung SF, Zee B, Chan AT: Treatmentofnasopharyngeal carcinoma with intensitymodulated radiotherapy: the Hong Kong experience. Int J Radiat Oncol Biol Phys 2004, 60:1440-1450.

35. Wolden SL, Chen WC, Pfister DG, Kraus DH, Berry SL, Zelefsky MJ: Intensity modulated radiation therapy (IMRT)for nasopharynx cancer: update of the Memorial Sloan-Ketteringexperience. Int J Radiat Oncol Biol Phys 2006, 64:57-62.

doi:10.1186/1471-2407-14-832

Cite this article as: Tian et al:: Nasopharyngeal carcinoma with paranasal sinus invasion: the prognostic significance and the evidence-based study basis of its T-staging category according to the AJCC staging system. BMC Cancer 2014 14:832.

\section{Submit your next manuscript to BioMed Central and take full advantage of:}

- Convenient online submission

- Thorough peer review

- No space constraints or color figure charges

- Immediate publication on acceptance

- Inclusion in PubMed, CAS, Scopus and Google Scholar

- Research which is freely available for redistribution

Submit your manuscript at www.biomedcentral.com/submit 